\title{
Application of cytokine-induced myeloid-derived suppressor cells mitigates murine pancreatic insulitis through inhibition of activated T cells
}

ching chuan hsieh ( $\square$ jeffrey570404@gmail.com )

Chang Gung Memorial Hospital Chiayi Branch https://orcid.org/0000-0001-8554-328X

Chun-Liang Lin

Chang Gung Medical College: Chang Gung University

Meihua Chiang

Chang Gung Memorial Hospital Chiayi Branch: Chiayi Chang Gung Memorial Hospital

\section{Yong-Lin Chang}

Chang Gung Memorial Hospital Chiayi Branch: Chiayi Chang Gung Memorial Hospital

Jie-Teng He

Chang Gung Memorial Hospital Chiayi Branch: Chiayi Chang Gung Memorial Hospital

Chien-Hui Hung

Chang Gung University

\section{Research Article}

Keywords: cytokine-induced myeloid-derived suppressor cells, pancreatic insulitis, immunotherapy

Posted Date: August 25th, 2021

DOl: https://doi.org/10.21203/rs.3.rs-829410/v1

License: (9) (i) This work is licensed under a Creative Commons Attribution 4.0 International License.

Read Full License 


\section{Abstract \\ Background}

Type 1 diabetes is an inflammatory state. Myeloid-derived suppressive cells (MDSCs) originate from immature myeloid cells and quickly expand to control host immunity during infection, inflammation, trauma, and cancer. Immunotherapy is a method of disease treatment that involves stimulating or suppressing an immune response. Although the spleens and blood of tumor-bearing mice are the most common MDSC source for immunotherapy, many safety precautions must be considered.

\section{Methods}

Cytokine-induced MDSCs were propagated from bone marrow cells cultured with mouse recombinant granulocyte-macrophage colony-stimulating factor (GM-CSF), interleukin (IL)-6, and IL-1 $\beta$. Arginase-1 and inducible NO synthase (iNOS) expressions of MDSCs were evaluated using quantitative reversetranscriptase PCR. Diabetes was diagnosed as a sustained blood sugar level $>350 \mathrm{mg} / \mathrm{dL}$. The immunized T cells harvested from the spleens of nonobese diabetic (NOD) mice cotransplanted with cMDSCs were injected intravenously to the NOD mice with severe combined immune deficiency (SCID), twice a week for 5 consecutive weeks. Diabetes-free survival of NOD-SCID mice was measured. Fibronectin expressions of the renal glomerulus and insulin production of the pancreas were analyzed using immunohistochemistry studies.

\section{Results}

This study presents an ex vivo procedure to develop MDSCs from bone marrow cells propagated from GM-CSF, IL-6, and IL-1 $\beta$ cytokines expressing less costimulatory and more inhibitory surface markers as well as considerably high levels of the iNOS enzyme through the signal transducer and activator of transcription 1 (STAT1) signaling pathway. In addition, the MDSCs also displayed immature morphology and strong immunosuppression of T cell proliferation. The adoptive transfer of cMDSCs improved the hyperglycemic state in and prolonged the diabetes-free survival of NOD-SCID mice induced by reactive splenic T cells harvested from NOD mice. In addition, cMDSC use reduced fibronectin production in the renal glomeruli and improved pancreatic insulitis in the NOD-SCID mice.

\section{Conclusions}

The administration of CMDSCs propagated from GM-CSF, IL-6, and IL-1 $\beta$ cytokines provides an alternative immunotherapy protocol for the treatment of diabetic pancreatic insulitis and renal nephropathy.

\section{Background}


Type 1 diabetes (T1D) is characterized by chronic inflammatory response associated with the autoimmune destruction of insulin-producing beta cells in the pancreas [1]. Emerging evidence has revealed that autoreactive T cells play a crucial role in spontaneous pancreatic insulitis resulting in $\beta$ cell loss [2]. Complications of T1D include renal failure, retinopathy, ischemic heart disease, and chronic foot ulcers [3].

Myeloid-derived suppressive cells (MDSCs) originate from immature myeloid cells and quickly expand to regulate the host's immune response during cancer, trauma, infection, and some autoimmune disorders $[4,5]$. In mice, MDSCs are characterized by the coexpression of the myeloid lineage differentiation antigen Gr1 and CD11b [6]. In humans, MDSCs are broadly defined as CD14 ${ }^{-} \mathrm{CD} 11 \mathrm{~b}^{+}$cells or cells that express CD33 but lack the markers of mature myeloid and lymphoid cells and the MHC-class-II molecule HLA-DR $[7,8]$. The suppressive activity of MDSCs has been associated with arginase I, inducible nitric oxide synthase (iNOS) [9, 10], reactive oxygen species [11, 12], anti-inflammatory cytokines [5], regulatory $T$ cells $[13,14]$, and tumor-associated macrophages $[15,16]$.

Immunotherapy is a method of disease treatment that involves stimulating or suppressing an immune response and provides an alternative treatment protocol for patients with cancer, inflammation, and organ transplantation [17]. The anti-inflammatory and immunoregulatory properties of MDSCs are commonly used in immunotherapy [18]. The common protocol is to use MDSCs differentiated from peripheral blood mononuclear cells or bone marrow (BM) cells with high concentrations of granulocytemacrophage colony-stimulating factor (GM-CSF) by itself or in combination with other factors [19].

In this study, we propagated MDSCs from mouse BM cells with GM-CSF, interleukin (IL)-6, and IL-1 $\beta$ cytokines in vitro and characterized their functions. In addition, we examined the regulatory effects of cytokine-induced MDSCs (cMDSCs) on pancreatic insulitis and renal glomerulosclerosis in nonobese diabetic (NOD) mice and mice with severe combined immune deficiency (SCID).

\section{Methods}

\section{Mouse models}

Female BALB/C, C57BL/6 (B6), NOD, and NOD-SCID mice were supplied by the National Laboratory Animal Center (Taiwan). All animal experiments were approved by the Institutional Animal Care and Use Committee of Chang Gung Memorial Hospital (IACUC permit number: 2016032802) and were performed in accordance with the Animal Protection Law by the Council of Agriculture, Executive Yuan (Taiwan) and the National Research Council's Guide for the Care and Use of Laboratory Animals (USA).

\section{Culture of cytokine-induced myeloid-derived suppressor cells}

BM cells $\left(2 \times 10^{6}\right.$ cells/well) from the tibias and femurs of the BALB/c mice were cultured in Roswell Park Memorial Institute 1640 medium containing $10 \%$ fetal bovine serum with mouse recombinant GM-CSF 
(10 ng/mL; R\&D Systems, Minneapolis, MN, USA), IL-6 (10 ng/mL; R\&D Systems), and IL-1 $\beta$ (10 ng/mL; R\&D Systems) for 7 days. Cells coexpressing CD11b and Gr-1 were considered cMDSCs.

\section{cMDSCs cotransplanted with immunized splenic T cells into NOD-SCID mice}

T cells ( $\mathrm{CD}^{+}, 2 \times 10^{7}$ cells) harvested from the spleens of NOD mice were injected intravenously to the NOD-SCID mice with or without the CMDSCs $\left(1 \times 10^{7}\right.$ cells), twice a week for 5 consecutive weeks. The total adoptive cells volume for intravenous injection were 20 million cells in the group of $T$ cells alone and 30 million cells in the group of T cells cotransplanted cMDSCs within 100 ul every time. Blood sugar levels and body weight were measured weekly. Diabetes was diagnosed as a sustained blood sugar level $>350 \mathrm{mg} / \mathrm{dL}$ by using a OneTouch UltraEasy monitor (Johnson and Johnson, New Brunswick, NJ, USA).

\section{Flow cytometry analysis}

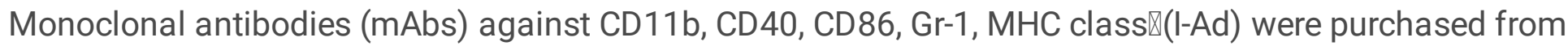
BD PharMingen (San Diego, CA, USA), and $m A$ bs against B7-H1 were purchased from eBioscience (San Diego, CA, USA). For CFSE labeling, splenic T cells $\left(10^{7}\right.$ cells $\left./ \mathrm{mL}\right)$ from B6 were incubated with $0.5 \mu \mathrm{M}$ of CFSE (Invitrogen, San Diego, CA, USA) for $10 \mathrm{~min}$ at room temperature. Flow analyses were performed using a BD FACSCanto II flow cytometer (BD Bioscience, Franklin Lakes, NJ, USA).

\section{Quantitative reverse transcription polymerase chain reaction}

Total RNA was extracted by using a RNeasy Mini Kit (Qiagen, Valencia, CA, USA). RNA samples were converted into cDNA by using the RevertAid First Strand cDNA Synthesis Kit (Thermo Fisher, Waltham, $M A, U S A)$. The following primers were used for the quantitative polymerase chain reaction (qPCR): arginase 1: forward CACGG CAGTG GCTTT AACCT and reverse TGGCG CATTC ACAGT CACTT; iNOS: forward TGGCC ACCTT GTTC AG CTACG and reverse GCCAA GGCCA AACAC AGCAT AC; fibronectin: forward GCTCA GCAAA TCGTG CAGC and reverse CTAGG TAGGT CCGTT CCCACT; STAT1: forward CTGAATATTTCCCTCCTGGG and reverse TCCCGTACAGATGTCCATGAT; STAT3: forward CTTGTCTACCTCTACCCCGACAT and reverse GATCCATGTCAAACGTGAGCG. mRNAs were measured using the CFX96 Touch Real Time PCR Detection System (Bio-Rad Laboratories, Inc., Hercules, CA, USA) in duplicate and normalized to $18 \mathrm{~S}$ mRNA.

\section{Immunofluorescence staining and confocal microscopy}

Tissue samples of pancreatic islets were embedded in optimal cutting temperature compound and snapfrozen in liquid nitrogen. Tissue sections $(4 \mu \mathrm{m})$ were fixed in an ethanol-acetic acid fixative solution for 2-10 min and stained with anti-insulin antibody (1:100, SC-8033, Santa Cruz Biotechnology, Inc., Santa Cruz, CA, USA) with secondary antibody (1:400, Fab'2 Donkey anti Mouse IgG-AF594, Jackson ImmunoResearch Inc. West Grove, PA, USA), DAPI (Invitrogen), and Alexa Fluor 488 Phalloidin (Invitrogen) overnight at $4{ }^{\circ} \mathrm{C}$ in a humidified chamber. After three washes, slides were stained with DAPI and mounted 
with ProLong Gold mounting medium (Invitrogen). Confocal imaging was performed by using a Leica SP5 II confocal microscope.

\section{Immunohistochemistry}

Extracellular matrix expression of the renal cortex in the cryostat sections was identified through fluorescent staining by using specific antifibronectin (Abcam), anti-insulin (Santa Cruz), anti-CD3 (BD PharMingen), and anti-Gr1 (BD Biosciences) antibodies after permeabilization with $0.05 \%$ saponin buffer by using a Vectastain Elite ABC kit (Vector Lab, Inc., Burlingame, CA, USA) for an immunoperoxidase procedure. The slides were developed using AEC chromogen substrate and counterstained with hematoxylin. Isotype- and species-matched irrelevant antibodies served as controls.

\section{Blood and urine analysis}

Blood glucose was determined using a OneTouch UltraEasy monitor (Johnson and Johnson). Blood and urine were collected for examination of serum creatinine, $\mathrm{HbA} 1 \mathrm{c}$, and urinary protein when the mice were sacrificed. Serum creatinine, $\mathrm{HbA} 1 \mathrm{c}$, and urinary protein levels were determined by using a Labospect 008 (Hitachi, Tokyo, Japan).

\section{Statistical analysis}

The statistical analysis was performed using Student's t test for independent samples, with $P<.05$ indicating significance. A log-rank test was performed to analyze diabetes-free survival. All data, means, and standard deviations were calculated and graphed in Microsoft Excel 2016 version (Microsoft, Redmond, WA, USA).

\section{Results}

\section{Spontaneous pancreatic insulitis and renal glomerulosclerosis in NOD mice}

The blood sugar levels of the NOD mice at 7 weeks old was $126.0 \pm 4.0 \mathrm{mg} / \mathrm{dL}$ and gradually increased to higher than $350 \mathrm{mg} / \mathrm{dL}$ at the age of 10 weeks $(358.0 \pm 2.5 \mathrm{mg} / \mathrm{dL})$. The blood sugar levels of the BALB/c mice remained steady at approximately $116.0 \pm 2.6 \mathrm{mg} / \mathrm{dL}$ throughout the experiment (Fig. 1a, left panel). Decreases in the body weight of the NOD mice were associated with hyperglycemia, whereas slight increases in the body weight of the BALB/c mice were not associated with blood sugar levels (Fig. 1a, right panel).

Autoimmune diabetes spontaneously developed in the NOD mice from autoreactive T cells. Hematoxylin and eosin staining of the pancreatic islets of the NOD mice at 14 weeks old revealed that, compared with the pancreatic islets of the BALB/c mice, the islet cell mass of the NOD mice was lost and the islets were surrounded by a leukocytic infiltration (Fig. 1b, upper left panel). A substantial decrease in insulin 
production due to beta-cell mass loss was observed in the NOD mice through immunofluorescence staining (Fig. 1b, upper right and lower right panels). Blood glycosylated hemoglobin (HbA1c) levels (\%) in the NOD mice were higher than those in the BALB/c mice (Fig. 1b, lower left panel).

Nephropathy is a major complication of diabetes. Extracellular matrix (ECM) accumulation in the renal parenchyma is a key etiological element in the pathogenesis of diabetic nephropathy. In our study, fibronectin, a major component of ECM, was more highly expressed in the renal glomerulus of the NOD mice than in that of the BALB/c mice (Fig. 1 c, upper panels). Serum creatinine ( $0.81 \pm 0.10$ vs $0.33 \pm 0.10$ $\mathrm{mg} / \mathrm{dL})$ and urinary protein $(196.67 \pm 16.0 \mathrm{vs} 31.0 \pm 3.6 \mathrm{mg} / \mathrm{dL})$ levels were noticeably higher in the NOD mice than in the BALB/c mice (Fig. 1c, lower panels).

Diabetes occurred in the NOD mice as a result of spontaneous insulitis, a leukocytic infiltration within pancreatic islets, leading to the loss of pancreatic beta cells. Renal dysfunction occurred after fibronectin accumulated in the renal glomerulus, resulting in glomerulosclerosis during hyperglycemia.

\section{MDSCs differentiated from GM-CSF, IL-6, and IL-1 $\beta$ cytokines displayed potent immunosuppressive activity}

MDSCs are propagated from myeloid progenitor cells and exhibit immunosuppressive activity rather than immunostimulatory properties. To ensure the strength of the immunosuppressive properties of the MDSCs, we developed several ex vivo procedures to differentiate MDSCs from BM cells through the GMCSF cytokine by itself or in combination with other molecules. The results displayed in Fig. 2a revealed that the yield ratio of MDSCs was higher in the G + IL- $6+$ IL-1 $\beta$ group (92.6\%) than in the GM-CSF group (54.6\%), the G + IL-6 group (78\%), and the G + IL-1 $\beta$ group (73\%; Fig. 2a, upper left panel). Among the four groups, the production of MDSCs was also highest in the $\mathrm{G}+\mathrm{IL}-6+\mathrm{IL}-1 \beta$ group (Fig. 2a, right panel). The MDSCs propagated from the G $+\mathrm{IL}-6+\mathrm{IL}-1 \beta$ group were round and exhibited a small amount of cytoplasmic projection as well as a large nuclear-cytoplasmic ratio, revealing the immature state of immune cells (Fig. 2a, lower left panel). The MDSCs in the G + IL-6 + IL-1 $\beta$ group expressed less costimulatory CD40, CD86, and MHC class $囚$ and more inhibitory B7H1 among the four groups, indicating that MDSCs display a greater capacity to suppress the immune response under the influence of three mixed cytokines (Fig. 2b).

In general, MDSCs obtain their immunosuppressive properties by expressing high levels of arginase 1 and inducible NOS through the signal transducer and activator of transcription (STAT) family of transcription factors. In this study, MDSCs were propagated from GM-CSF, IL-6, and IL-1 $\beta$ cytokines that expressed considerably high levels of STAT1 but not STAT3 transcription factors in all four groups (Fig. 2c, upper panel). Moreover, the MDSCs noticeably upregulated the expression of the iNOS enzyme rather than the production of arginase 1 under the influence of the GM-CSF, IL-6, and IL-1 $\beta$ cytokines (Fig. 2c, lower panel). These results demonstrated that the MDSCs developed from the GM-CSF, IL-6, and IL-1 $\beta$ cytokines exuded their immunoregulatory properties through the iNOS enzyme via the STAT1 signaling pathway. 
To examine the effect of the MDSCs on adaptive immunity, the MDSCs were cocultured with allogeneic spleen T cells stained with carboxyfluorescein succinimidyl ester (CFSE), and the proliferation rate of the T cells was examined. The MDSCs from the G + IL-6 + IL-1 $\beta$ group exhibited the strongest ability to suppress the $T$ cell proliferation rate with a dose-dependent effect in comparison with the other groups (Fig. 2d). The production of IFNy, a proinflammatory cytokine, was less secreted from $\mathrm{T}$ cells stimulated by GM-CSF, IL-6 and IL-1 $\beta$ molecules (Fig. 2e).

In conclusion, the MDSCs differentiated from the GM-CSF, IL-6, and IL-1 $\beta$ cytokines had immature morphology, upregulated the production of iNOS through STAT1 signaling, and exhibited high immunosuppression of T cell proliferation.

\section{Application of cMDSCs improved pancreatic insulitis and prolonged diabetes-free survival}

Autoreactive T cells prompt T1D development by causing severe destruction of pancreatic $\beta$ cells. Studies have noted that MDSCs employ their immunoregulatory properties to suppress $T$ cell proliferation. To determine whether CMDSCs propagated from GM-CSF, IL-6, and IL-1 $\beta$ cytokines influence the properties of T cells that cause T1D diabetes in vivo, T cells were harvested from the spleens of NOD mice and intravenously administered to the NOD-SCID mice by themselves or cotransplanted with CMDSCs twice a week for 5 consecutive weeks (Fig. 3a, upper panel). Blood sugar levels in the group treated with T cells alone were the highest among the three groups, whereas the blood sugar levels decreased in the group treated with T cells cotransplanted with cMDSCs. The body weight of the NOD-SCID mice was lowest in the group treated with $T$ cells alone, whereas the group treated with the combination of $T$ cells and cMDSCs exhibited a slight decrease in body weight compared with the untreated group (Fig. 3a, lower panel). Treatment with the cMDSCs cotransplanted with T cells considerably improved the diabetes-free survival of the NOD-SCID mice in comparison with the group treated with T cells alone (Fig. 3b). As shown in Fig. 3c, the population of MDSCs in the T cells + cMDSCs group was higher in the pancreas compared with the other two groups.

Nephropathy causes the accumulation ECM proteins in the mesangial interstitial space and is a detrimental effect of diabetes. Treatment with $\mathrm{T}$ cells considerably increased the fibronectin production in the renal glomerulus of the NOD-SCID mice, and administration of cMDSCs improved fibronectin expression in the renal glomerulus (Fig. 3d, upper panel). The levels of serum creatinine and urinary protein noticeably increased in the NOD-SCID mice after treatment with T cells compared with the untreated mice, whereas the application of cMDSCs improved renal function and proteinuria in the NODSCID mice (Fig. 3d, lower panel).

A deficiency of insulin production in pancreatic islets is the main etiology of T1D. The data in the upper panel of Fig. 3e demonstrate that the production of insulin in the NOD-SCID mice treated with the T cells harvested from NOD mice was the lowest among the three groups, whereas insulin production increased after cotransplantation with cMDSCs. HbA1c levels, the gold standard for monitoring chronic high blood sugar, considerably increased in the group treated with $\mathrm{T}$ cells alone compared with the untreated group (Fig. 3e, lower panel). The treatment of cMDSCs cotransplanted with T cells reduced HbA1c levels 
compared with the treatment of T cells alone. The upper panel of Fig. $3 f$ indicates that the number of T cells $\left(\mathrm{CD}^{+}\right)$within the pancreatic islets was higher in the NOD-SCID mice treated with T cells alone or with cotransplanted cMDSCs than that in the untreated mice. The decrease in the number of T cells was correlated with the increase in the number of CMDSCs in the pancreatic islets of the NOD-SCID mice (Fig. 3f, lower panel).

In summary, the application of cMDSCs propagated from GM-CSF, IL-6, and IL-1 $\beta$ cytokines can improve renal function and increase insulin secretion from pancreatic islets, thereby prolonging the diabetes-free survival of NOD-SCID mice after treatment with T cells.

\section{Discussion}

MDSCs originate from the myeloid-cell lineage and contain a diverse population of precursors of myeloid cells and myeloid-cell progenitors. These immature myeloid cells quickly expand to control host immunity during infection, inflammation, trauma, and cancer $[4,5]$. The anti-inflammatory and immunoregulatory properties of MDSCs include increasing the expression of reactive oxygen species [11, 12], arginase 1, iNOS $[9,10]$, anti-inflammatory cytokines [5], regulatory T cells $[13,14]$, macrophages associated with tumors [15, 16], proangiogenic factors [20], and the impairment of natural killer cell cytotoxicity [21]. In a prior study, we demonstrated that MDSCs induced by hepatic stellate cells (HSCs) exhibited high immunoregulatory properties and prolonged the survival of mice with cotransplanted islet allografts through the induction of effector $T$ cell apoptosis and the generation of regulatory $T$ cells [22]. The soluble molecule complement component 3 (C3) secreted from the HSCs was involved in the MDSCs' expression of an inhibitory immune response [23]. In addition, the MDSCs produced inhibitory enzymes through the IL- 6 signaling pathway to reduce effector T cell immunity and promoted tumor progression within the tumor microenvironment [24-27]. The role of MDSCs in inflammatory and infectious disorders has been studied $[28,29]$.

Immunotherapy is a method of treating a disease through the induction, augmentation, or inhibition of an immune response. Adoptive cell immunotherapy is an inspiring area of cancer therapy that commenced in the 1980s. Chimeric antigen receptor (CAR) T cell immunotherapy for the treatment of B cell hematologic neoplasms is one of the most hopeful examples. Alternatively, to widespread CAR T cells, Natural Killer (NK) cells or macrophages may be genetically transduced to generate CAR NK cells or CAR $M$ cells that now rapidly expand to non-cancer fields such as infectious disorders and autoimmune diseases $[30,31]$. Although the spleens and blood of tumor-bearing mice are the most commonly used sources of MDSCs for immunotherapy, many safety precautions must be considered [18]. The most widely used ex vivo protocol to propagate MDSCs from BM cells or peripheral blood mononuclear cells involves high concentrations of GM-CSF alone or in combination with other factors [19]. Our previous research revealed that IL- 6 cytokine can increase the propagation of MDSCs within tumor microenvironments $[24,26,27]$. We reported that IL-1 $\beta$ cytokine derived from pancreatic cancer promoted the population of M2 macrophages, MDSCs, $C D 1 \mathrm{~d}^{\text {hi }} \mathrm{CD}^{+}$regulatory $\mathrm{B}$ cells, and Th17 cells resulted in immunoregulation [32]. Lechner et al. reported that the MDSCs propagated in vitro from human peripheral 
blood mononuclear cells from GM-CSF and IL- 6 exhibited the strongest ability to inhibit T cell proliferation among seven cytokine mixtures [33]. In the present study, we developed an ex vivo procedure to develop MDSCs from BM cells propagated from GM-CSF, IL-6, and IL-1 $\beta$ cytokines that expressed less costimulatory and more inhibitory surface markers as well as considerably high levels of the iNOS enzyme through the STAT1 signaling pathway. In addition, the MDSCs differentiated from the GM-CSF, IL6 , and IL-1 $\beta$ cytokines exhibited immature morphology and strong immunosuppression of T cell proliferation.

T1D is an insulin-dependent disorder characterized by a chronic inflammatory state against insulin autoantigens, leading to the eventual destruction of pancreatic $\beta$ cells [1]. Autoreactive T cells cause the loss of $\beta$ cells associated with pancreatic insulitis [2]. NOD mouse models have been widely used and have been a crucial part of research on the mechanism of autoimmunity and T1D [34-36]. In this study, diabetes onset, that is, a blood sugar level $>350 \mathrm{mg} / \mathrm{dL}$, began at 10 weeks of age in the NOD mice, accompanied by a decrease in body weight. Four weeks later, pancreatic islets were surrounded by a leukocytic infiltrate, leading to loss of islet cell mass. The effects of spontaneous pancreatic insulitis resulted in decreased insulin production and elevated levels of HbA1c in the blood. Nephropathy is a complication of diabetes that is the most common cause of renal failure worldwide [37]. Fibronectin, a major component of ECM, was highly expressed and accumulated in the renal glomeruli of the NOD mice. Glomerulosclerosis was caused by excessive fibronectin accumulation. The NOD mice exhibited impairments in renal function caused by elevated serum creatinine and proteinuria levels. These manifestations in the kidneys of the NOD mice were similar to those of the mice with streptozotocininduced diabetes, which involved chemically-induced pancreatic insulitis [38].

To determine whether the cMDSCs developed from the GM-CSF, IL-6, and IL- $1 \beta$ cytokines regulating T cell activity influenced the pathogenesis of T1D in mice, T cells were harvested from the spleens of the NOD mice and administered by themselves or cotransplanted with cMDSCs into the NOD-SCID mice. The results of this study indicate that cMDSC administration improved the hyperglycemic state of the NODSCID mice induced by the reactive splenic T cells harvested from the NOD mice and prolonged their diabetes-free survival. Cytokine-induced MDSCs exhibited the immunoregulatory effect of inhibiting effector T cell function, improved pancreatic insulitis, and increased insulin production. In addition, the application of cMDSCs reduced fibronectin production in the renal glomeruli and improved renal function in the NOD-SCID mice. Our previous study also reported that the anti-inflammatory properties of cMDSCs are crucial for improving renal glomerulosclerosis in mice with chemically-induced diabetes [38].

\section{Conclusion}

The administration of CMDSCs propagated from GM-CSF, IL-6, and IL-1 $\beta$ cytokines prolonged the diabetes-free survival of diabetic mice by way of inhibition of activated T cells. In addition, CMDSC use reduced fibronectin production in the renal glomeruli and improved pancreatic insulitis in the NOD-SCID mice. The present study provides an alternative immunotherapy protocol for the treatment of diabetic pancreatic insulitis and renal nephropathy. 


\section{Abbreviations}

BM: Bone marrow

C3: Component 3

CFSE: Carboxyfluorescein succinimidyl ester

cMDSC: Cytokine-induced MDSC

ECM: Extracellular matrix

GM-CSF: Granulocyte-macrophage colony-stimulating factor

HbA1c: Glycosylated hemoglobin

HSC: Hepatic stellate cell

iNOS: Inducible nitric oxide synthase

IL: Interleukin

MDSC: Myeloid-derived suppressor cell

NOD: Nonobese diabetic

SCID: Severe combined immune deficiency

STAT: Signal transducer and activator of transcription

T1D: Type 1 diabetes

\section{Declarations}

Acknowledgments: The authors express their gratitude to the Expensive Advanced Instrument Core Laboratory in the Department of Medical Research and Development, Chiayi Chang Gung Memorial Hospital, for allowing us to use their BD FACSCanto II flow cytometer, BD FACSAria Fusion cell sorter, and Leica SF5 II confocal microscope.

Funding: The work was supported by the Ministry of Science and Technology (Taiwan) under grant number 106-2314-B-182A-104-MY2 and by Chang Gung Memorial Hospital under grant number CMRPG6F0413 (all to C.C.H.).

\section{Availability of data and materials}

The authors declare that the datasets supporting the results of this study are included within the article. 
Author Contributions: Conceptualization, C.-C.H. and C.-L.L.; Methodology, C.-C.H.; Software, J.-T.H.; Validation, J.-T.H., M.C., and Y.-L.C.; Formal Analysis, M.C.; Investigation, C.-C.H.; Resources, C.-C.H.; Data Curation, M.C.; Writing-Original Draft Preparation, C.-C.H.; Writing-Review and Editing, C.-C.H.; Supervision, C.-L.L. and C.-H.H.; Project administration, Y.-L.C.; Funding Acquisition, C.-C.H. All authors read and approved the final manuscript.

Ethics Approval: All animal experiments were approved by the Institutional Animal Care and Use Committee of Chang Gung Memorial Hospital (IACUC permit number: 2016032802) and performed in accordance with the Animal Protection Law of the Council of Agriculture, Executive Yuan (Taiwan) and the National Research Council's Guide for the Care and Use of Laboratory Animals (US).

\section{Consent for publication}

Not applicable

Conflicts of Interest: The authors declare no conflicts of interest.

\section{References}

1. Atkinson MA, Eisenbarth GS, Michels AW. Type 1 diabetes. Lancet. 2014;383:69-82. [doi: 10.1016/S0140-6736(13)60591-7] [ PMID: 23890997].

2. Burrack AL, Martinov T, Fife BT. T Cell-Mediated Beta Cell Destruction: Autoimmunity and Alloimmunity in the Context of Type 1 Diabetes. Front Endocrinol (Lausanne). 2017;8:343. [doi: 10.3389/fendo.2017.00343] [PMCID: PMC5723426].

3. Atkinson MA, Maclaren NK. The pathogenesis of insulin-dependent diabetes mellitus. N Engl J Med. 1994;331:1428-36. [doi: 10.1056/NEJM199411243312107] [PMID: 7969282].

4. Gabrilovich DI, Nagaraj S. Myeloid-derived suppressor cells as regulators of the immune system. Nat Rev Immunol. 2009;9:162-74. [doi: 10.1038/nri2506] [PMID: 19197294].

5. Gabrilovich DI. Myeloid-Derived Suppressor Cells. Cancer Immunol Res. 2017;5:3-8. [doi: 10.1158/2326-6066.CIR-16-0297] [PMID: 28052991].

6. Kusmartsev S, Nefedova Y, Yoder D, Gabrilovich DI. Antigen-specific inhibition of CD8 + T cell response by immature myeloid cells in cancer is mediated by reactive oxygen species. $\mathrm{J}$ Immunol. 2004;172:989-99. [doi: 10.4049/jimmunol.172.2.989] [PMID: 14707072].

7. Ochoa AC, Zea AH, Hernandez C, Rodriguez PC. Arginase, prostaglandins, and myeloid-derived suppressor cells in renal cell carcinoma. Clin Cancer Res. 2007;13:721s-6s. [doi: 10.1158/10780432.CCR-06-2197] [PMID: 17255300].

8. Almand B, Clark JI, Nikitina E, van Beynen J, English NR, Knight SC, Carbone DP, Gabrilovich DI. Increased production of immature myeloid cells in cancer patients. A mechanism of immunosuppression in cancer. J Immunol. 2001;166:678-89. [doi: 10.4049/jimmunol.166.1.678] [PMID: 11123353]. 
9. Rodriguez PC, Hernandez CP, Quiceno D, Dubinett SM, Zabaleta J, Ochoa JB, Gilbert J, Ochoa AC. Arginase I in myeloid suppressor cells is induced by COX-2 in lung carcinoma. J Exp Med. 2005;202:931-9. [doi: 10.1084/jem.20050715] [PMID: 16186186].

10. Rodriguez PC, Ochoa AC. Arginine regulation by myeloid derived suppressor cells and tolerance in cancer: mechanisms and therapeutic perspectives. Immunol Rev. 2008;222:180-91. [doi: 10.1111/j.1600-065X.2008.00608.x] [PMID: 18364002].

11. Kusmartsev S, Nagaraj S, Gabrilovich DI. Tumor-associated CD8 + T cell tolerance induced by bone marrow-derived immature myeloid cells. J Immunol. 2005;175:4583-92. 10.4049/jimmunol.175.7.4583] [PMID: 16177103]. [doi:.

12. Agostinelli E, Seiler N. Non-irradiation-derived reactive oxygen species (ROS) and cancer: therapeutic implications. Amino Acids. 2006;31:341-55. [doi: 10.1007/s00726-005-0271-8] [PMID: 16680401].

13. Yang R, Cai Z, Zhang Y, Yutzy WH, Roby KF, Roden RB. CD80 in immune suppression by mouse ovarian carcinoma-associated Gr-1 + CD11b + myeloid cells. Cancer Res. 2006;66:6807-15. [doi: 10.1158/0008-5472.CAN-05-3755] [PMID: 16818658].

14. Huang B, Pan PY, Li Q, Sato Al, Levy DE, Bromberg J, Divino CM, Chen SH. Gr-1 + CD115 + immature myeloid suppressor cells mediate the development of tumorinduced $\mathrm{T}$ regulatory cells and T-cell anergy in tumor-bearing host. Cancer Res. 2006;66:1123-31. [doi: 10.1158/0008-5472.CAN-05-1299] [PMID: 16424049].

15. Ostrand-Rosenberg S, Fenselau C. Myeloid-Derived Suppressor Cells: Immune-Suppressive Cells That Impair Antitumor Immunity and Are Sculpted by Their Environment. J Immunol. 2018;200:422-31. [doi: 10.4049/jimmunol.1701019] [PMID: 29311384].

16. Ruffell B, Coussens LM. Macrophages and therapeutic resistance in cancer. Cancer Cell. 2015;27:462-72. [doi: 10.1016/j.ccell.2015.02.015] [PMID: 25858805].

17. Law AMK, Valdes-Mora F, Gallego-Ortega D. Myeloid-Derived Suppressor Cells as a Therapeutic Target for Cancer. Cells. 2020;9:561. [doi: 10.3390/cells9030561] [PMID: 32121014].

18. Corzo CA, Condamine T, Lu L, Cotter MJ, Youn JI, Cheng P, Cho HI, Celis E, Quiceno DG, Padhya T, McCaffrey TV, McCaffrey JC, Gabrilovich DI. HIF-1a regulates function and differentiation of myeloidderived suppressor cells in the tumor microenvironment. J Exp Med. 2010;207:2439-53. [doi: 10.1084/jem.20100587] [PMID: 20876310].

19. Lutz MB, Kukutsch NA, Menges M, Rössner S, Schuler G. Culture of bone marrow cells in GM-CSF plus high doses of lipopolysaccharide generates exclusively immature dendritic cells which induce alloantigen-specific CD4 T cell anergy in vitro. Eur J Immunol. 2000;30:1048-52. [doi: 10.1002/(SICI)1521-4141(200004)30:4<1048::AID-IMMU1048>3.0.CO;2-W] [PMID: 10760792]

20. Boelte KC, Gordy LE, Joyce S, Thompson MA, Yang L, Lin PC. Rgs2 mediates pro-angiogenic function of myeloid derived suppressor cells in the tumor microenvironment via upregulation of MCP-1. PLoS One. 2011;6:e18534. [doi: 10.1371/journal.pone.0018534] [PMID: 21494556].

21. Elkabets M, Ribeiro VS, Dinarello CA, Ostrand-Rosenberg S, Di Santo JP, Apte RN, Vosshenrich CA. IL$1 \beta$ regulates a novel myeloid-derived suppressor cell subset that impairs NK cell development and 
function. Eur J Immunol. 2010;40:3347-57. [doi: 10.1002/eji.201041037] [PMID: 21110318].

22. Chou HS, Hsieh CC, Yang HR, Wang L, Arakawa Y, Brown K, Wu Q, Lin F, Peters M, Fung JJ, Lu L, Qian $\mathrm{S}$. Hepatic stellate cells regulate immune response by way of induction of myeloid suppressor cells in mice. Hepatology. 2011;53:1007-19. [doi: 10.1002/hep.24162] [PMID: 21374665].

23. Hsieh CC, Chou HS, Yang HR, Lin F, Bhatt S, Qin J, Wang L, Fung JJ, Qian S, Lu L. The role of complement component 3 (C3) in differentiation of myeloid-derived suppressor cells. Blood. 2013;121:1760-8. [doi: 10.1182/blood-2012-06-440214] [PMID: 23299310].

24. Wu CT, Hsieh CC, Lin CC, Chen WC, Hong JH, Chen MF. Significance of IL-6 in the transition of hormone-resistant prostate cancer and the induction of myeloid-derived suppressor cells. J Mol Med. 2012;90:1343-55. [doi: 10.1007/s00109-012-0916-x] [PMID: 22660275].

25. Chen MF, Hsieh CC, Chen WC, Lai CH. Role of interleukin- 6 in the radiation response of liver tumors. Int J Radiat Oncol Biol Phys. 2012;84:e621-30. [doi: 10.1016/j.jjrobp.2012.07.2360] [PMID: 22975618].

26. Chen PT, Hsieh CC, Wu CT, Yen TC, Lin PY, Chen WC, Chen MF. 1a,25-Dihydroxyvitamin D3 Inhibits Esophageal Squamous Cell Carcinoma Progression by Reducing IL6 Signaling. Mol Cancer Ther. 2015;14:1365-75. doi:10.1158/1535-7163.MCT-14-0952] [PMID: 25824337].

27. Hsieh CC, Hung CH, Chiang M, Tsai YC, He JT. Hepatic Stellate Cells Enhance Liver Cancer Progression by Inducing Myeloid-Derived Suppressor Cells through Interleukin-6 Signaling. Int J Mol Sci. 2019;20:5079. [doi: 10.3390/ijms20205079] [PMID: 31614930].

28. Lin WY, Hsieh CC, Yang TY, Chen ML, Huang LY, Lin YP, Chang PJ, Levin RM, Wei YH. Transient increase in circulating myeloid-derived suppressor cells after partial bladder outlet obstruction. $J$ Urol. 2014;192:1569-73. [doi: 10.1016/j.juro.2014.05.045] [PMID: 24840536].

29. Peng KT, Hsieh CC, Huang TY, Chen PC, Shih HN, Lee MS, Chang PJ. Staphylococcus aureus biofilm elicits the expansion, activation and polarization of myeloid-derived suppressor cells in vivo and in vitro. PLoS One. 2017;12:e0183271. [doi: 10.1371/journal.pone.0183271] [PMID: 28813499].

30. Titov A, Zmievskaya E, Ganeeva I, Valiullina A, Petukhov A, Rakhmatullina A, Miftakhova R, Fainshtein M, Rizvanov A, Bulatov E. Adoptive Immunotherapy beyond CAR T-Cells. Cancers (Basel). 2021;11:743. [doi: 10.3390/cancers13040743.PMID: 33670139].

31. Zmievskaya E, Valiullina A, Ganeeva I, Petukhov A, Rizvanov A, Bulatov E. Application of CAR-T Cell Therapy beyond Oncology: Autoimmune Diseases and Viral Infections. Biomedicines. 2021;9:59. [doi: 10.3390/biomedicines9010059] [PMID: 33435454].

32. Das S, Shapiro B, Vucic EA, Vogt S, Bar-Sagi D. Tumor Cell-Derived IL1 $\beta$ Promotes Desmoplasia and Immune Suppression in Pancreatic Cancer. Cancer Res. 2020;80:1088-101. [doi: 10.1158/00085472.CAN-19-2080] [PMID: 31915130].

33. Lechner MG, Liebertz DJ, Epstein AL. Characterization of Cytokine-Induced Myeloid-Derived Suppressor Cells from Normal Human Peripheral Blood Mononuclear Cells. J Immunol. 2010;185:2273-84. [doi: 10.4049/jimmunol.1000901] [PMID: 20644162]. 
34. Chen YG, Mathews CE, Driver JP. The Role of NOD Mice in Type 1 Diabetes Research: Lessons from the Past and Recommendations for the Future. Front Endocrinol (Lausanne). 2018;9:51. [doi: 10.3389/fendo.2018.00051] [PMID: 29527189].

35. Melanitou E, Devendra D, Liu E, Miao D, Eisenbarth GS. Early and quantal (by litter) expression of insulin autoantibodies in the nonobese diabetic mice predict early diabetes onset. $\mathrm{J}$ Immunol. 2004;173:6603-10. [doi: 10.4049/jimmunol.173.11.6603] [ PMID: 15557150].

36. Al-Awar A, Kupai K, Veszelka M, Szűcs G, Attieh Z, Murlasits Z, Török S, Pósa A, Varga C. Experimental Diabetes Mellitus in Different Animal Models. J Diabetes Res. 2016;2016:9051426. [doi: 10.1155/2016/9051426] [PMID: 27595114].

37. Schieppati A, Remuzzi G. Chronic renal disease as a public health problem: epidemiology, social, and economic implications. Kidney Int. 2005;98(Suppl.):7-10. [doi: 10.1111/j.1523-1755.2005.09801.x] [PMID: 16108976].

38. Hsieh CC, Lin CL, He JT, Chiang M, Wang Y, Tsai YC, Hung CH, Chang PJ. Administration of cytokineinduced myeloid-derived suppressor cells ameliorates renal fibrosis in diabetic mice. Stem Cell Res Ther. 2018;9:183. [doi: 10.1186/s13287-018-0915-0.PMID: 29973247] [PMID: 29973247].

\section{Figures}
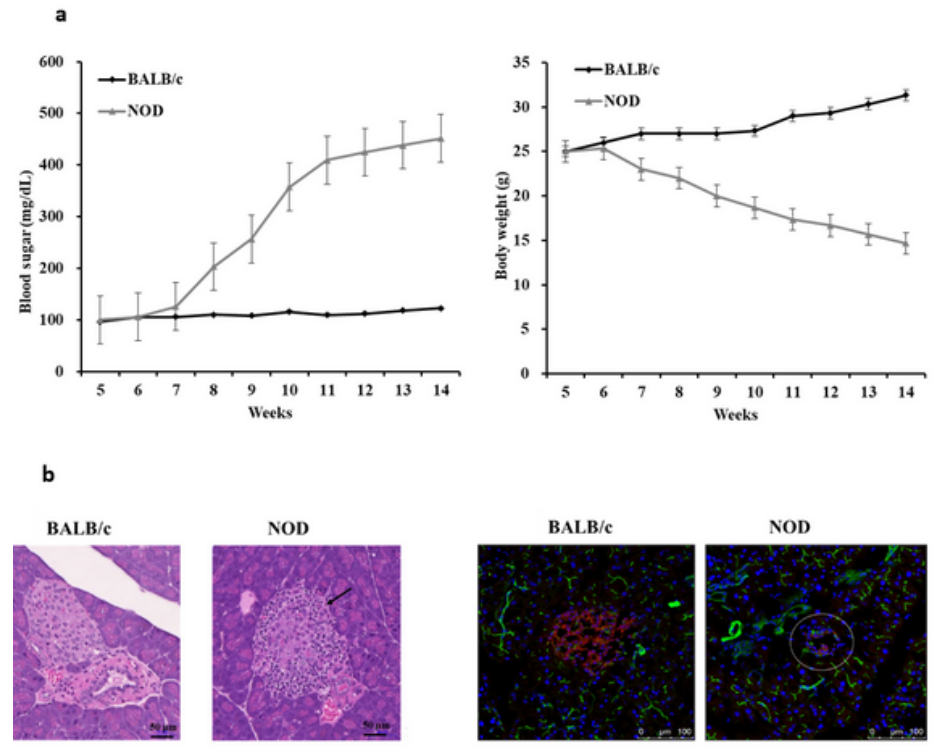

NOD
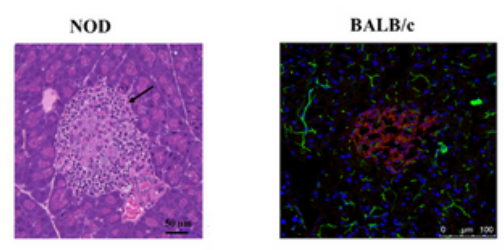

NOD
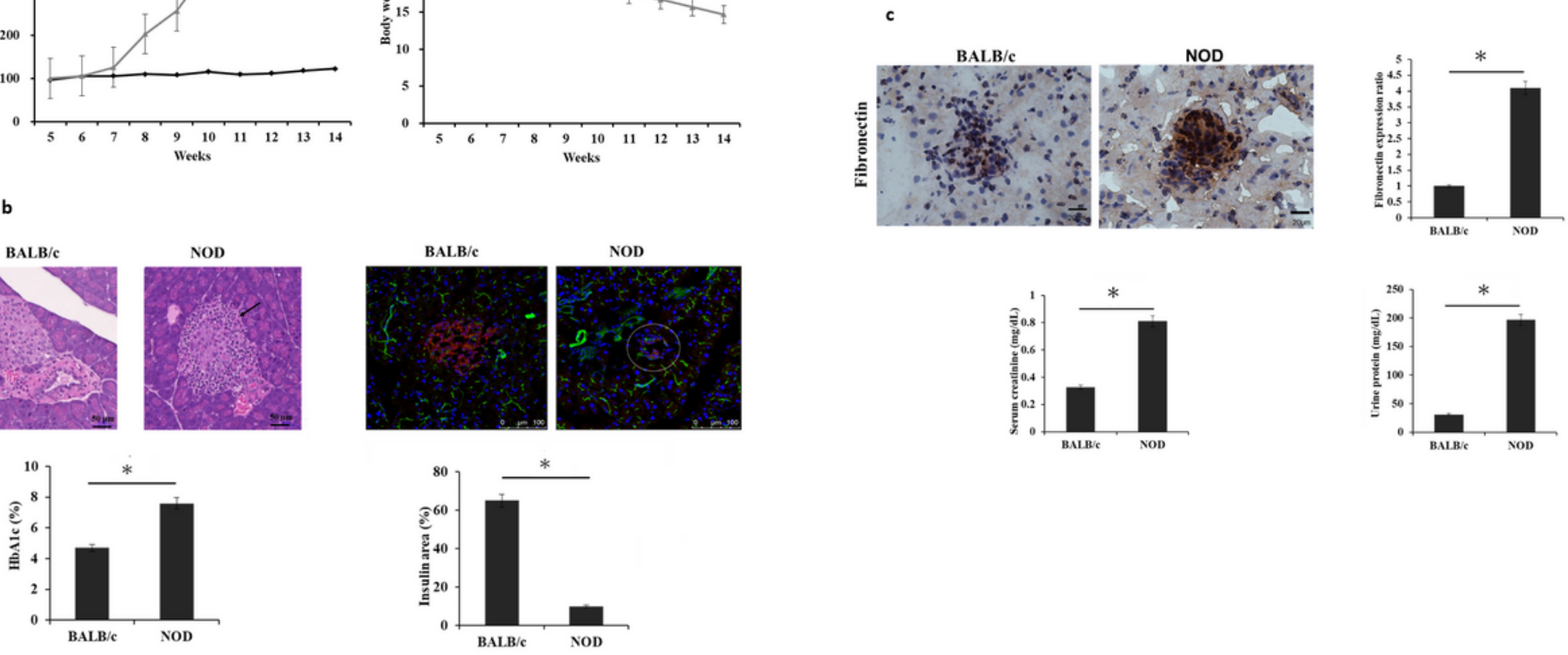

Figure 1

Expression of pancreatic and renal functions in nonobese diabetic (NOD) mice. a Blood sugar levels (left panel) and body weight (right panel) were measured once per week for the 5-week-old subgroups of 
BALB/c and NOD mice. Mice in each group were sacrificed on week 14. Diabetes was diagnosed as a sustained blood glucose level $>350 \mathrm{mg} / \mathrm{dL}$. b Pancreas histology for 14-week-old BALB/c and NOD mice stained with hematoxylin and eosin. BALB/c mice exhibited normal pancreatic islets surrounded by an exocrine portion. NOD mice exhibited islet cell destruction with the presence of leukocytes (arrowhead, $400 \times$ magnification; upper left panel). Cryostat sections of the pancreatic islets of the BALB/c and the NOD mice were stained with anti-insulin monoclonal antibody (mAb, red) and evaluated under a fluorescence microscope (400× magnification; upper right panel). Bar graphs present the blood glycosylated hemoglobin (HbA1c) levels (\%) and the insulin area of the pancreatic islets (\%) from the BALB/c and the NOD mice (lower panel, ${ }^{*} \mathrm{P}<.05$ ). c Cryostat sections of kidney histochemically stained with anti-fibronectin mAb (upper left panel, brown; 400× magnification) and examined using a microscope. The ratio of fibronectin expression within the renal glomeruli of the two groups was quantified (upper right panel, ${ }^{*} \mathrm{P}<.05$ ). Bar graphs display the levels of serum creatinine and urinary protein collected from the two groups at the 14 weeks old (lower panel, $* \mathrm{P}<.05$ ). Data are representative of three separate experiments. 

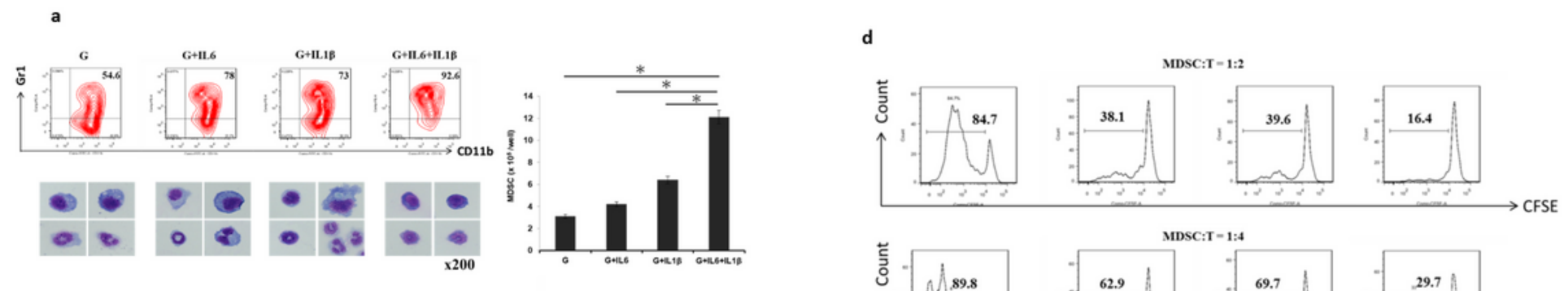

b
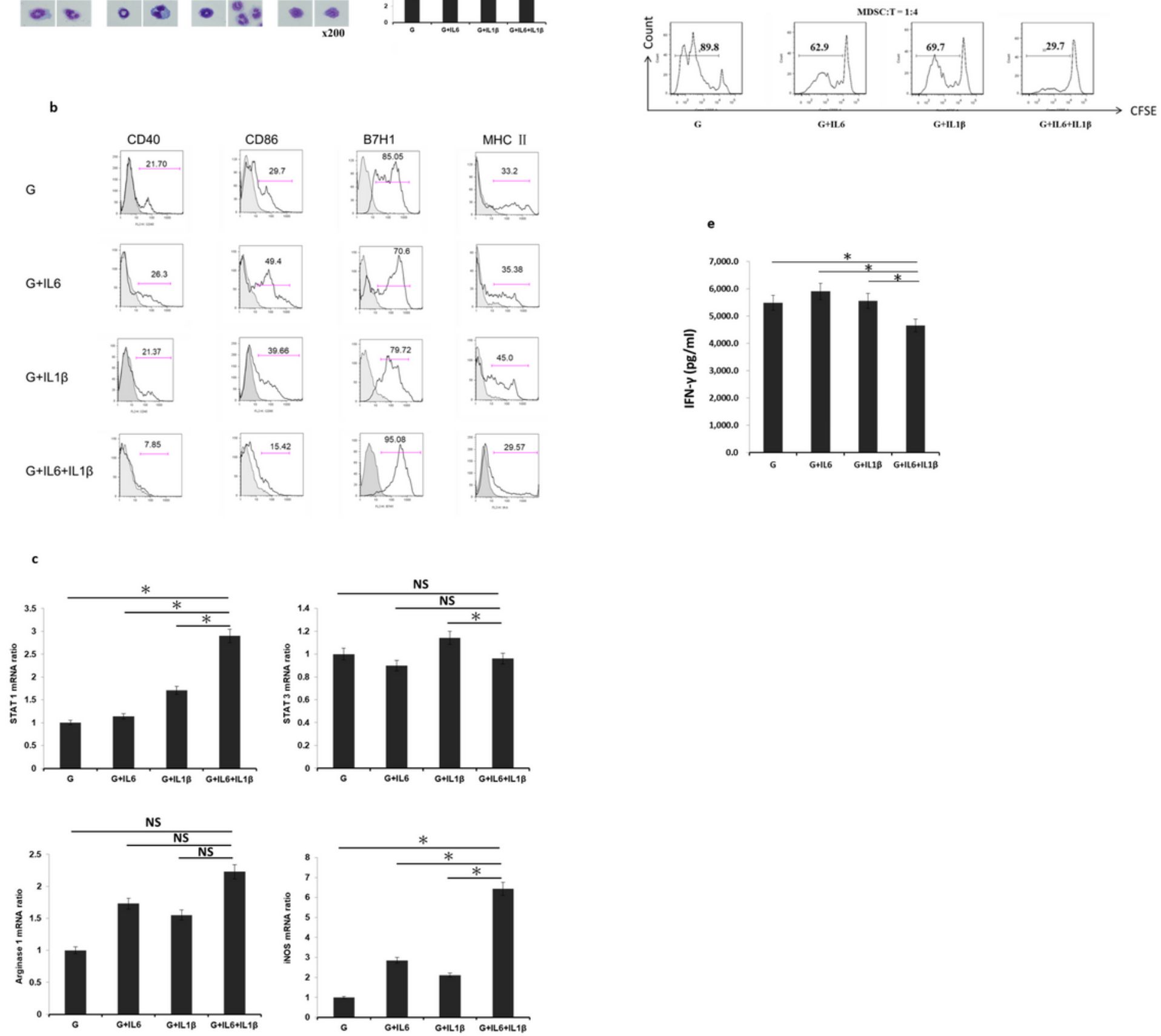

Figure 2

Characterization of cytokine-induced myeloid-derived suppressive cells (MDSCs). a MDSCs were propagated from bone marrow $(\mathrm{BM})$ cells $(2 \times 106$ cells/well), harvested from the tibias and femurs of the BALB/C mice, cultured with mouse recombinant granulocyte-macrophage colony-stimulating factor (GM-CSF; $10 \mathrm{ng} / \mathrm{mL})$, interleukin (IL)-6 (10 ng/mL), and IL-1 $\beta(10 \mathrm{ng} / \mathrm{mL})$ for 7 days. MDSCs were diagnosed as double positive for $\mathrm{CD} 11 \mathrm{~b}$ and $\mathrm{Gr}-1$. The results are displayed as a histogram in which the 
numbers correspond to the percentage of positive cells. The MDSCs were stained with Giemsa for morphology examination (200x magnification). The bar graph displays the yield of the MDSCs for each group ( ${ }^{*} \mathrm{P}<.05$ ). b Surface markers of MDSCs stained with specific mAbs against CD40, CD86, B7H1, $\mathrm{MHC}$ class $\otimes$. Numbers indicate percentage of positive cells. c Expression of the signal transducer and activator of transcription (STAT) 1, STAT3, arginase 1, and inducible nitric oxide synthase (iNOS) mRNA from the MDSCs of each group were determined using a quantitative polymerase chain reaction $\left({ }^{\star} \mathrm{P}<.05\right.$; NS: not significant). d Antigen-presenting activity of MDSCs. The MDSCs were cultured with carboxyfluorescein diacetate succinimidyl ester (CFSE)-labeled B6 spleen T cells at a 1:2 or 1:4 ratio for 3 days. Proliferative response was determined through CFSE dilution. e The levels of IFNY from T cells analyzed from supernatant of MDSC-stimulated T cells through ELISA $\left({ }^{*} \mathrm{P}<.05\right)$. Data are representative of three separate experiments. 

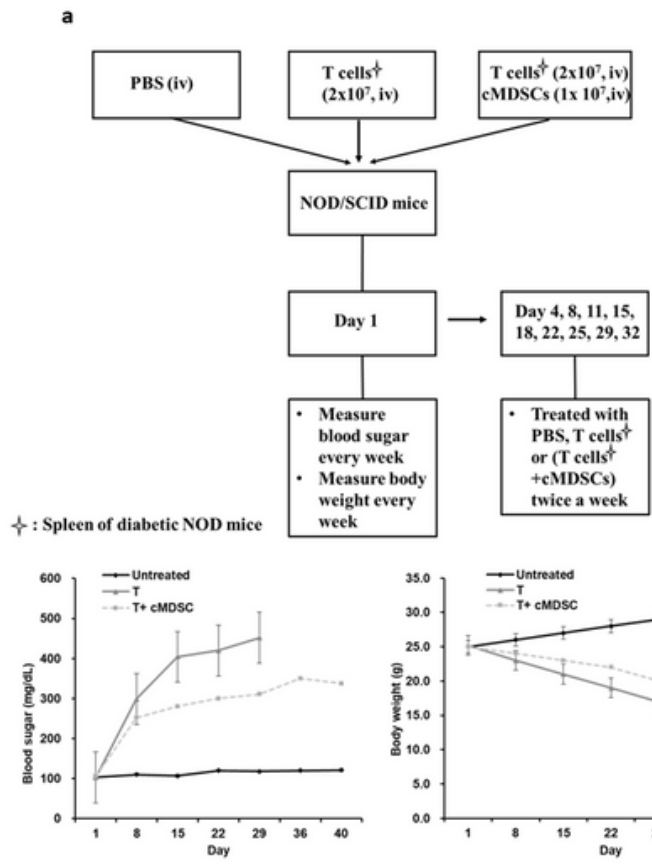

d
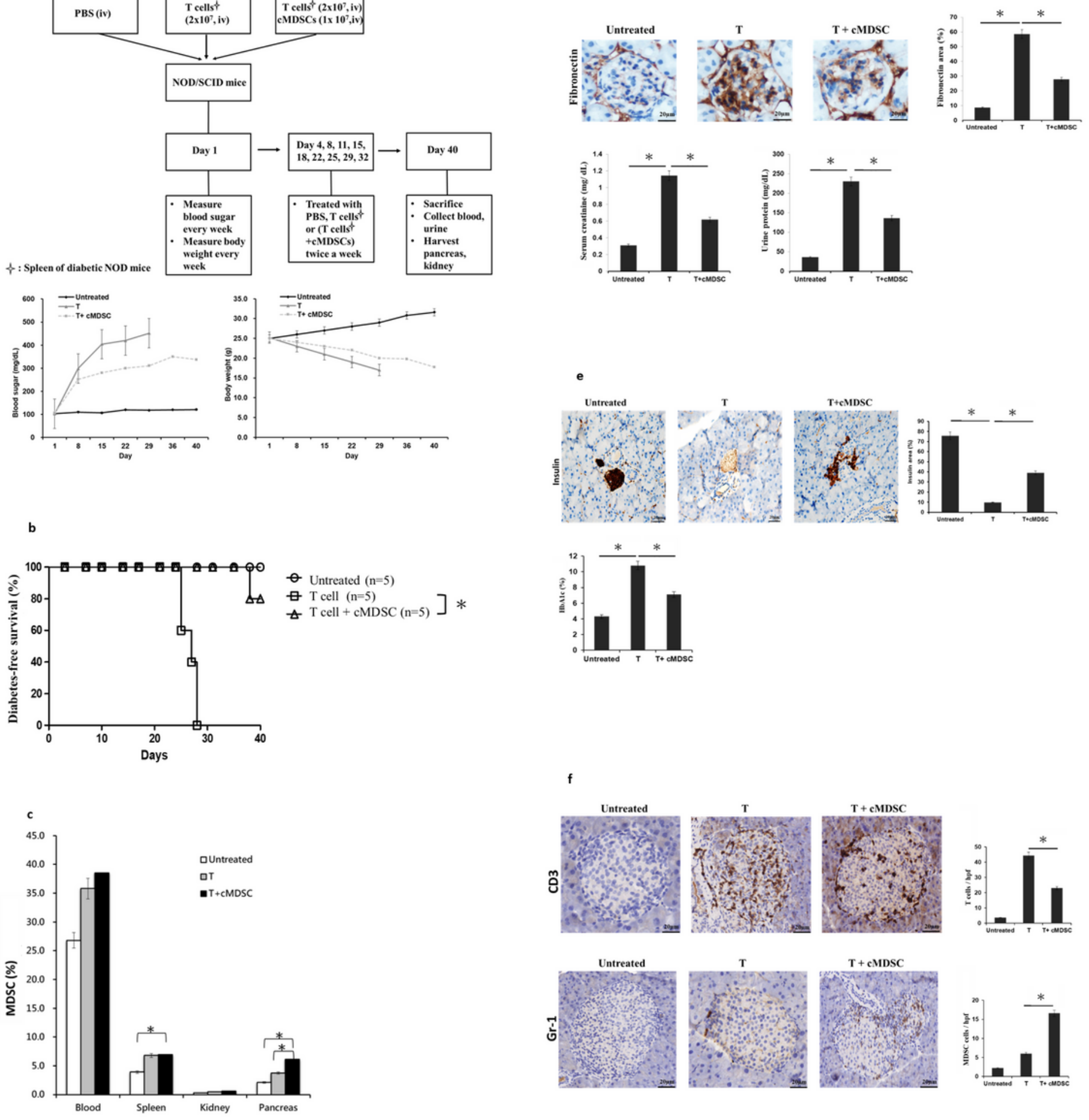

Figure 3

Application of cMDSCs to prolong the diabetes-free survival of NOD-SCID mice in vivo. a T cells (CD3+, 2 $\times 107$ cells) harvested from the spleens of NOD mice were injected intravenously into NOD-SCID mice with or without cMDSCs $(1 \times 107$ cells) twice a week for 5 consecutive weeks. The control group was treated with phosphate buffered saline (PBS). Blood sugar levels and body weight (lower panel) were measured every week. The mice were sacrificed on day 40 , and blood, urine, spleens, and pancreases 
were harvested for examination. b Diabetes-free survival of NOD-SCID mice after treatment with T cells alone, T cells cotransplanted with cMDSCs, or PBS. Diabetes was diagnosed as a sustained blood sugar level $>350 \mathrm{mg} / \mathrm{dL}\left({ }^{*} \mathrm{P}<.05\right)$. c MDSC ratios in blood, spleen, kidney, and pancreas of three groups compared. Isolated cells were two-color stained with specific mAbs against CD11b and Gr-1 for flow analyses ( $\left.{ }^{*} \mathrm{P}<.05\right)$. $d$ Kidney cryostat sections histochemically stained with antifibronectin (brown, 400x magnification) were examined under a microscope. The area of fibronectin expression within the glomeruli of the three groups was quantified $\left({ }^{*} \mathrm{P}<.05\right)$. Serum creatinine and urinary protein levels of the three groups were examined and compared $\left({ }^{*} P<.05\right)$. e Cryostat sections of the pancreas were histochemically stained with anti-insulin antibody (brown, 100x magnification) and examined under a microscope. The area of insulin secretion within the pancreatic islets of the three groups was quantified. $\mathrm{HbA1c}$ levels $(\%)$ in the blood were measured and compared $\left({ }^{*} \mathrm{P}<.05\right)$. $f$ The number of $T$ cells and MDSCs in the pancreatic islets was determined through histochemical staining with anti-CD3 or anti-Gr-1 mAb (brown, 400x magnification) and examination under a microscope. Bar graphs illustrate positive T cells and MDSC counts in a total of 10 high-power fields (400x magnification) randomly selected in each section $\left({ }^{*} \mathrm{P}<.05\right)$. Data are representative of three separate experiments. 